\title{
DISEÑO DE UNA RED FTTH PARA EL DISTRITO GREGORIO ALBARRACÍN LANCHIPA, TACNA
}

DESIGN OF FTTH NETWORK TO GREGORIO ALBARRACIN LANCHIPA DISTRICT, TACNA

Ernesto Antonio Rios De La Cruz ${ }^{1}$ Huido Quino Huanacuni ${ }^{2}$ Hugo Javier Rivera Herrera ${ }^{3}$

Información del artículo: Recibido: 25/05/2020 Aceptado: 18/06/2020

1,2 Bachiller en Ingeniería Electrónica. Email: ernesto .mast...pas@hotmail.com, hquino@gmail.com

${ }^{3}$ Docente en la Facultad de Ingeniería, Universidad Privada de Tacna. E-mail; hjrivera.upt@gmail.com

Vol. 2, N 1.

INGENIERÍA

INVESTIGA Enero - Junio del 2020. 


\section{Resumen}

El objetivo de la investigación fue diseñar una red de acceso FTTH para distribuir servicios de banda ancha en el distrito Gregorio Albarracín Lanchipa, en la provincia de Tacna. Se describen las características técnicas y bondades de la tecnología FTTH con el estándar GPON. Con el presente trabajo de investigación se pretende plantear una solución al problema de acceso de última milla a los servicios de telecomunicaciones del distrito, para lo cual se seleccionaron 3034 usuarios o suscriptores ubicados en las asociaciones de vivienda de las pampas de Viñani. Esta red de planta externa consta de la arquitectura de red de distribución, red de acceso y red de terminación además cuenta con la red de alimentación desde el proveedor de internet. Se realizó un mapeo para recopilar información referente a la ubicación de la red de postes de distribución eléctrica y así conocer la ubicación del recorrido cable de fibra óptica ADSS en el distrito, ubicación de los divisores ópticos y equipamiento a utilizar. Con la información recopilada se realizó el cálculo de presupuesto óptico, se determinó el ancho de banda requerido y el equipamiento tanto activo como pasivo.

Palabras claves: FTTH; GPON; Red de distribución; Red de Acceso; Presupuesto óptico,

\section{Abstract}

The objective of this research was design an access network with FTTH technology to distribute broadband services in Gregorio Albarracín Lanchipa district, in Tacna. The technical characteristics and benefits of FTTH technology with the GPON standard are described. This research work aims to propose a solution to the problem of last mile access to the district's telecommunications services, 3034 users or subscribers located in the housing associations of the Viñani pampas are selected. This external plant network consists of the distribution network, access network and termination network architecture, as well as the supply network from the internet provider. A mapping was carried out to collect information regarding the location of the network of electricity distribution poles and thus to know the location of the ADSS fiber optic cable route in the district, location of the optical dividers and equipment to be used. With the information collected, the calculation of the optical budget was made, the required bandwidth and the active and passive equipment to be used were determined.

Keywords: FTTH; GPON; Distribution Network; Access Network; Optical Budget. 


\section{Introducción}

En el ámbito mundial de las telecomunicaciones, los proveedores de servicios están transformando sus redes desplegadas de cobre, utilizando nuevas tecnologías a través de fibra óptica, para poder brindar y soportar mayor tasa de bits, velocidad, ancho de banda y estabilidad. Una de las tecnologías de mayor penetración es la denominada "Fibra hasta el hogar" o FTTH por sus siglas en inglés "Fiber to the home". FTTH permite brindar una alta velocidad de comunicación a usuarios como hogares, empresas, colegios, hospitales, etc. Así, FTTH permite brindar los servicios de telemedicina, educación, administración negocios en la nube, domótica, internet de las cosas, video bajo demanda, entretenimiento y todo servicio que requiera alta velocidad de conexión.

A medida que el uso de un mayor ancho de banda se ha masificado, las plataformas de servidores y almacenamiento de datos han migrado a la nube, logrando eficiencia económica y escalabilidad, pero a su vez convirtiéndose en un punto crítico, debido a que es de vital importancia mantener la disponibilidad, integridad, eficiencia del servicio. Debido a esto una red de fibra óptica permite integrar todo servicio que requiera disponibilidad, integridad y eficiencia sea fiable y seguro, así, una tecnología como FTTH llega a ser una opción viable.

En nuestro país actualmente nos encontramos a la espera de un despliegue descentralizado de redes FTTH para que hogares, empresas, colegios y hospitales adquieran servicios de banda ancha.

\section{Objetivo}

Diseñar una red FTTH para distribuir servicios de banda ancha en el distrito Gregorio Albarracín Lanchipa, provincia Tacna.

\section{Metodología}

El presente trabajo de investigación es de tipo experimental. Para el levantamiento de la información se realizaron visitas de campo, a fin de determinar la distribución física de los potenciales usuarios del servicio y la topología de la red, para plantear una solución al problema de acceso de última milla a los servicios de telecomunicaciones del distrito Gregorio Albarracín Lanchipa, provincia de Tacna.

La red FTTH, bajo el estándar de Red Óptica pasiva con capacidad de Gigabit (GPON) de la ITU-T, compromete diferentes factores incluyendo tamaño, costo y escalabilidad, teniendo en cuenta que no existe un modelo de una red de acceso FTTH estandarizado, ya que la viabilidad del proyecto depende fuertemente de la densidad de suscriptores (suscriptores/Km2) y la estructura (calles, avenidas, edificios, casas, etc).

La red considerada en este proyecto provee los servicios de internet, Television por Protocolo de Internet (IPTV) y Telefonía IP, por lo que la gestión del ancho de banda viene a ser un factor importante, ya que este aspecto garantiza la calidad y la satisfacción de los usuarios con 
respecto a los servicios propuestos. En tal sentido, es de vital importancia conocer el total de ancho de banda de cada uno de los servicios ofrecidos y la relación con los divisores ópticos de primer y segundo nivel. La figura 1 muestra el procedimiento seguido para el diseño de la red FTTH.

\section{Figura 1}

Diagrama de flujo del diseño de una red de acceso FTTH.

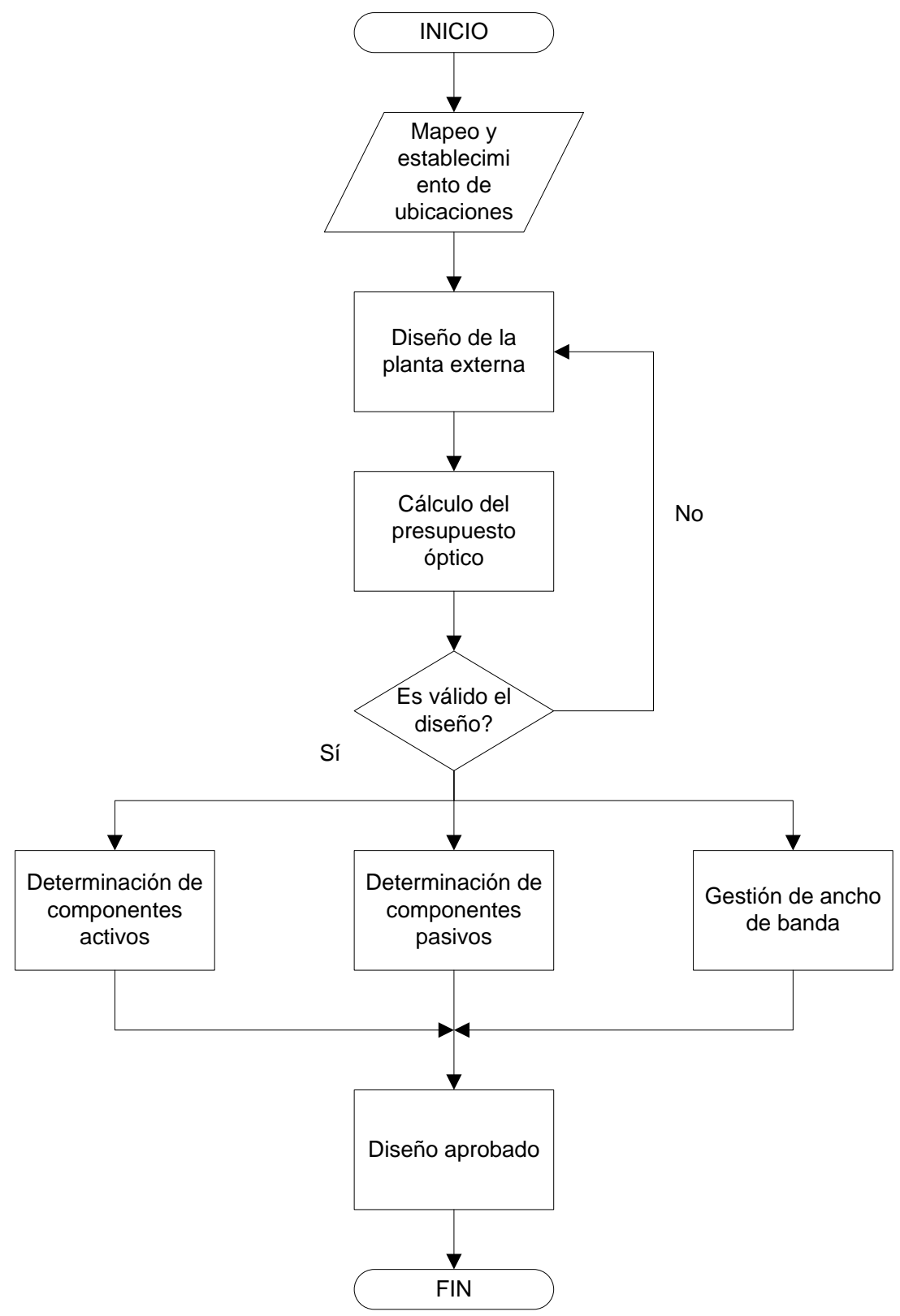

Nota: Modificado de Al-Quzwini (2014).

\section{Resultados}

A partir de la recopilación de la información geográfica de la zona sobre la cual se trabajó, se seleccionó la cantidad de lotes por asociación de vivienda para diseñar la red de acceso. 
Figura 2

Delimitación del Sector 10 MDGAL.

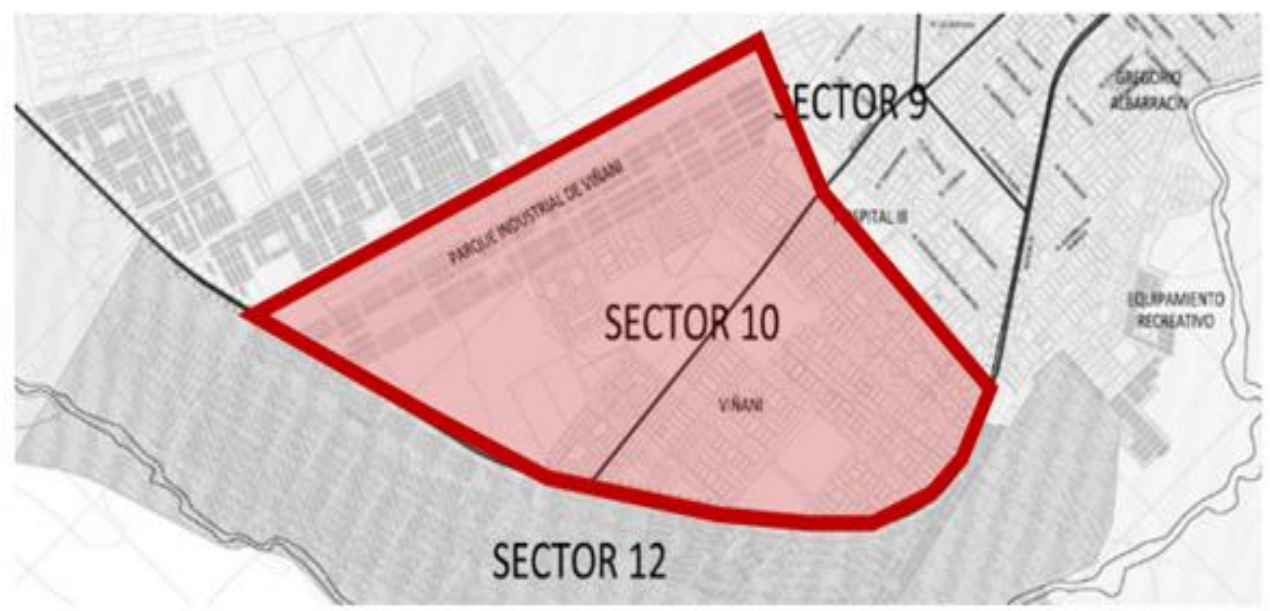

Nota. Elaboración equipo PAT-PDU 2014-2023 a partir del Catastro MDGAL

\section{Tabla 1}

Consolidado General de Viviendas por Asociación

\begin{tabular}{lc}
\hline Asociación de vivienda & Total Viviendas \\
\hline Ciudad de Paz & 666,00 \\
Paseo de los Heroes & 755,00 \\
Virgen de la Candelaria & 52,00 \\
Villa Colonial & 536,00 \\
Ciudad Futura & 140,00 \\
El Comerciante & 140,00 \\
La Joya & 129,00 \\
El Nazareno & 137,00 \\
Los Florales & 121,00 \\
La Perla & 145,00 \\
Ex Combatientes del Cenepa & 59,00 \\
Los Geranios & 154,00 \\
\hline Total de viviendas & 3034.00 \\
\hline
\end{tabular}

Nota. Elaboración Propia.

El cálculo del requerimiento de ancho de banda es determinante para la distribución y la cantidad de paquetes de ancho de banda a contratar para la redistribución de los servicios de banda ancha. Además, a partir de los resultados obtenidos, se definió la topología de red, la cantidad de divisores ópticos a usar y el número de puertos GPON necesarios para una cantidad definida de usuarios. 


\section{Tabla 2}

Requerimiento de Banda Ancha en Mbps

\begin{tabular}{lrr}
\hline \multirow{2}{*}{ Servicio } & \multicolumn{2}{c}{ Ancho de Banda } \\
\cline { 2 - 3 } & Subida & Bajada \\
\hline Internet & 2,00 & 20,00 \\
IPTV (3canales x 8Mbps) & 0,10 & 16,00 \\
Telefonía & 0,25 & 0,25 \\
\hline TOTAL & 2,35 & 36,25 \\
\hline
\end{tabular}

Nota: IPTV; Televisión por Protocolo de Internet. Elaboración propia.

La red troncal debe soportar la transmisión y recepción de grandes cantidades de datos, requiriendo un gran ancho de banda con altas velocidades. Estará soportada sobra la red de postes de distribución de energía eléctrica, para lo cual se realizó el levantamiento de información de la infraestructura instalada, con un total de 56 postes de red eléctrica. Parte desde la ubicación de la oficina central y ODF hasta la ubicación del punto de distribución que es la mufa 01, la cual permite realizar los empalmes y la redistribución de la red hasta la ubicación de los CDF de distribución donde están ubicados los divisores ópticos de primer nivel.

\section{Figura 3}



Nota. Fuente Google Earth.

La red de distribución y red de acceso se constituyeron a través de dos niveles de divisores ópticos. De acuerdo a las características del terreno y a los requerimientos de potencia, en el primer nivel se utilizó un divisor óptico 1:4 y en el segundo nivel, de 1:16, obteniendo un hilo de fibra óptica para 64 suscriptores asociados a un solo puerto GPON. 


\section{Figura 4}

Red de distribución

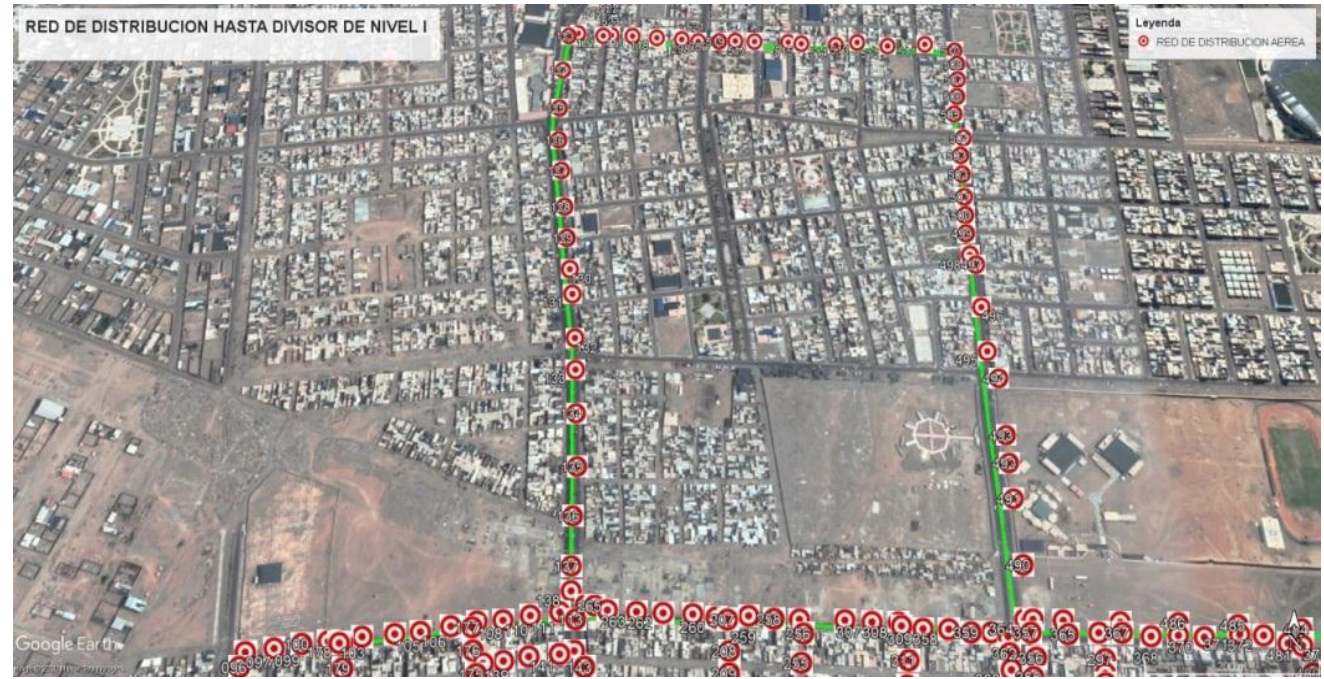

Nota. Fuente Google Earth.

La red FTTH se extiende desde el equipo terminal de línea óptica (OLT) ubicado en la oficina central y el equipo terminal de red óptico (ONT) ubicado en el local del usuario. Entre estos puntos de conexión se extiende una red de equipos ópticos, la distancia máxima entre estos equipos no supera la distancia lineal de $20 \mathrm{Km}$ para el tramo más distante del usuario, de esta forma se garantizó que la atenuación óptica total desde la OLT hasta la ONT no supere los $25 \mathrm{~dB}$, obteniendo un margen óptico aceptable.

\section{Figura 5}

Red de acceso.

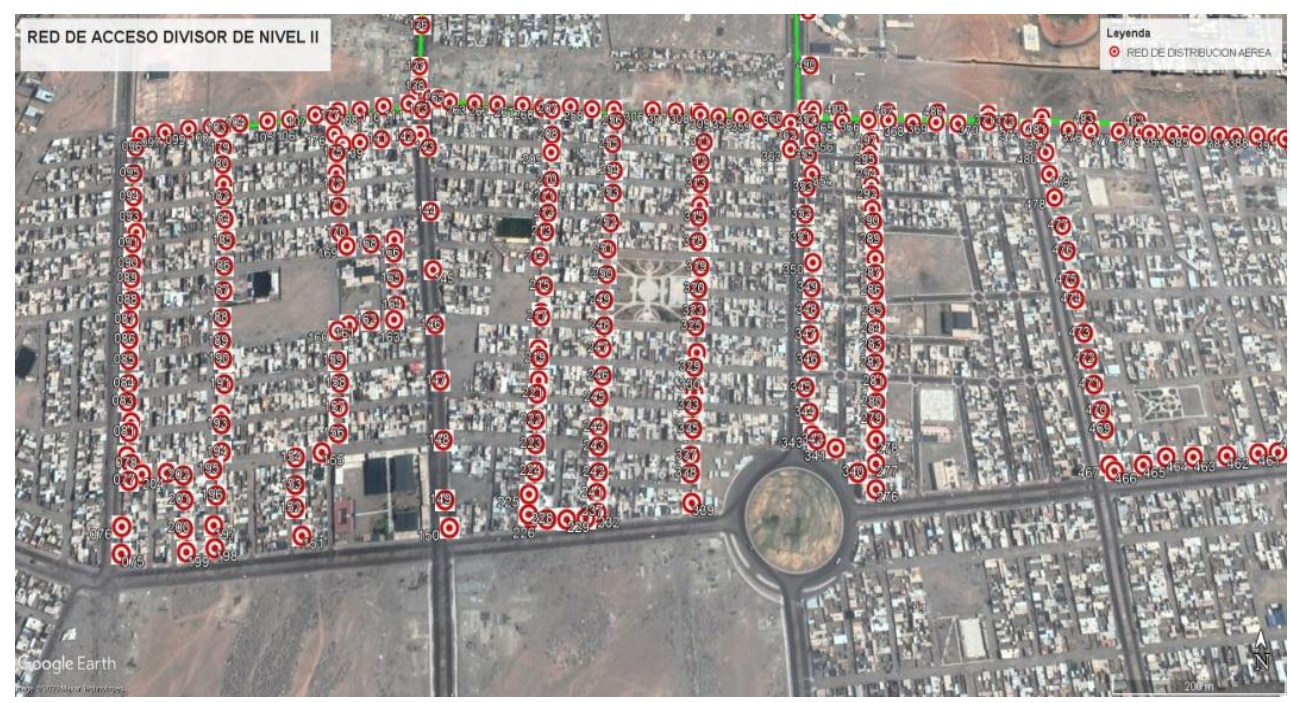

Nota. Fuente Google Earth.

La Figura 6 muestra la topología de la red FTTH diseñada, describiendo los niveles detallados para la red de distribución y de acceso. 


\section{Figura 6}

Topologia óptica de arquitectura de RED FTTH

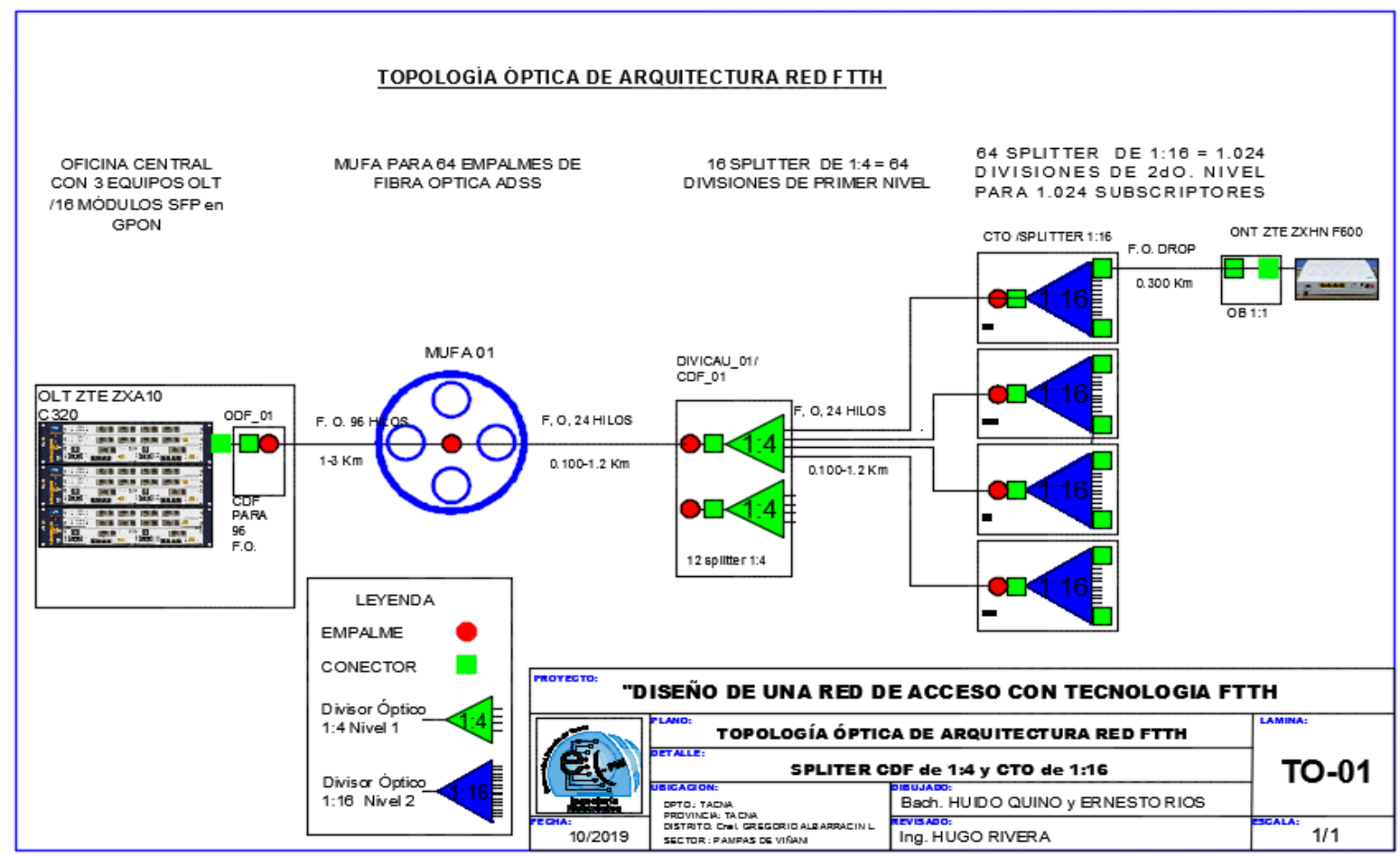

Nota. Elaboración propia.

Con la siguiente fórmula se realizó el cálculo del presupuesto óptico:

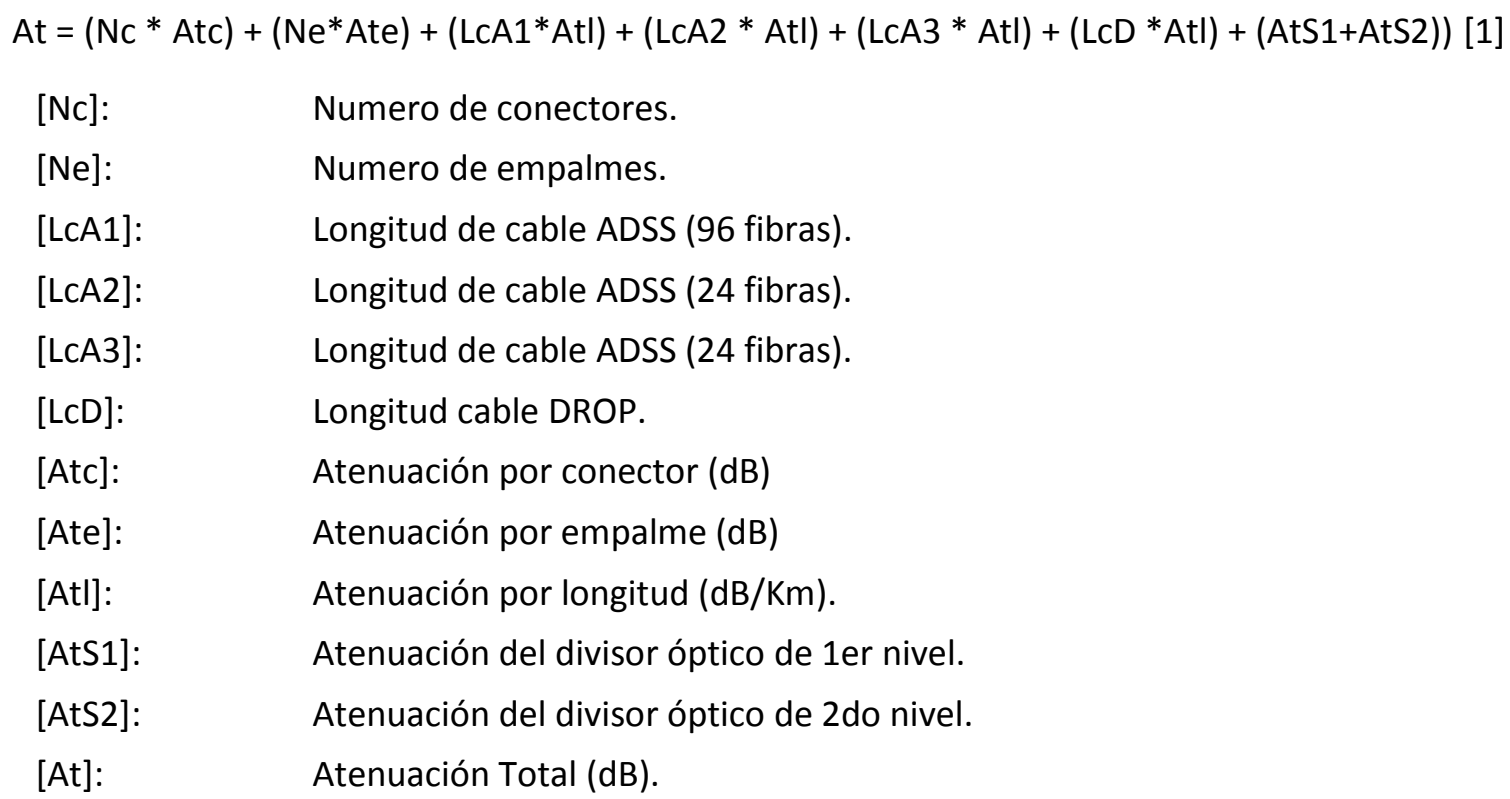

Con los equipos seleccionados se determina el margen de atenuación óptico para la OLT y la ONT conforme se muestra en las tablas 3 y 4 , considerando como pero caso el usuario más alejado de la OLT. 
Tabla 3

Determinacion de Margen de Atenuacion Óptico para OLT (peor caso)

\begin{tabular}{clcccc}
\hline Item & \multicolumn{1}{c}{ Parámetros } & Cantidad & Atenuación & Presupuesto & Unidad \\
\hline 1,1 & Potencia del transmisor TX & & 0,00 & $\mathrm{dBm}$ \\
1,2 & Sensibilidad del Receptor RX & & $-30,00$ & $\mathrm{dBm}$ \\
\hline $\mathrm{A}$ & Margen de potencia disponible del sistema & 30,00 & $\mathrm{~dB}$ \\
\hline 2,1 & Km. F. O. ADSS 96 HILOS & 1,021 & 0,33 & 0,34 & $\mathrm{~dB}$ \\
2,2 & SPLITER 1:4 & 1,000 & 7,26 & 7,26 & $\mathrm{~dB}$ \\
2,3 & Km. F.O. 24 HILOS & 1,231 & 0,33 & 0,40 & $\mathrm{~dB}$ \\
2,4 & SPLITER 1:16 & 1,000 & 13,08 & 13,08 & $\mathrm{~dB}$ \\
2,5 & Km. F.O. 24 HILOS & 0,753 & 0,33 & 0,25 & $\mathrm{~dB}$ \\
2,6 & Km. F.O. DROP 1 HILOS & 0,300 & 0,23 & 0,07 & $\mathrm{~dB}$ \\
2,7 & Conectores OLT-ONT & 8,000 & 0,20 & 1,60 & $\mathrm{~dB}$ \\
2,8 & Empalmes OLT-ONT & 5,000 & 0,10 & 2,00 & $\mathrm{~dB}$ \\
\hline $\mathrm{B}$ & Total de pérdida en atenuaciones & & 24,98 & $\mathrm{~dB}$ \\
\hline $\mathrm{C}=\mathrm{A}-\mathrm{B}$ & Margen de Enlace & & 5,02 & $\mathrm{~dB}$ \\
\hline
\end{tabular}

Nota. Elaboración propia.

Tabla 4

Determinacion de Margen de Atenuacion Óptico para ONT (peor caso)

\begin{tabular}{|c|c|c|c|c|c|}
\hline Item & Parámetros & Cantidad & Atenuación & Presupuesto & Unidad \\
\hline 1,1 & Potencia del transmisor TX & & & 0,50 & $\mathrm{dBm}$ \\
\hline 1,2 & Sensibilidad del Receptor RX & & & $-28,00$ & $\mathrm{dBm}$ \\
\hline A & Margen de potencia disponible del & stema & & 28,50 & $d B$ \\
\hline 2,1 & Km. F. O. ADSS 96 HILOS & 1,021 & 0,33 & 0,34 & $d B$ \\
\hline 2,2 & SPLITER 1:4 & 1,000 & 7,26 & 7,26 & $d B$ \\
\hline 2,3 & Km. F.O. 24 HILOS & 1,231 & 0,33 & 0,40 & $d B$ \\
\hline 2,4 & SPLITER 1:16 & 1,000 & 13,08 & 13,08 & $d B$ \\
\hline 2,5 & Km. F.O. 24 HILOS & 0,753 & 0,33 & 0,25 & $d B$ \\
\hline 2,6 & Km. F.O. DROP 1 HILOS & 0,300 & 0,23 & 0,07 & $d B$ \\
\hline 2,7 & Conectores OLT-ONT & 8,000 & 0,20 & 1,60 & $d B$ \\
\hline 2,8 & Empalmes OLT-ONT & 5,000 & 0,10 & 2,00 & $d B$ \\
\hline $\mathrm{B}$ & Total de perdida en atenuaciones & & & 24,98 & $d B$ \\
\hline$C=A-B$ & Margen de Enlace & & & 3,52 & $d B$ \\
\hline
\end{tabular}

Nota. Elaboración propia.

\section{Conclusión}

Se diseñó una red FTTH para distribuir servicios de banda ancha en el distrito Gregorio Albarracín Lanchipa de la provincia de Tacna, usando estándares técnicos aprobados por la ITU-T.

Se realizó un análisis técnico para implementar servicios Triple Play en banda ancha, empleando una red FTTH bajo el estándar GPON. 
Se calcularon y validaron los márgenes de atenuación óptico para todos los usuarios del servicio diseñado.

\section{Referencias Bibliográficas}

Al-Quzwini, M. (2014). Design and implementation of a Fiber to the Home acces network based on GPON. New Jersey: International Journal of Computer Applications.

Asensi Pla, J. S. (2011). DESIGN OF PASSIVE OPTICAL NETWORK. Valencia.

Axess Network. (s.f.). PLC Splitters.

Asensi Pla, J. S. (2011). DESIGN OF PASSIVE OPTICAL NETWORK. Valencia.

BANCO CENTRAL DE RESERVA DEL PERÚ. (s.f.). BCRPData. Obtenido de https://estadisticas.bcrp.gob.pe/estadisticas/series/anuales/resultados/PM05241PAPM05242PA-PM05243PA-PM05244PA-PM05245PA-PM05246PA-PM05247PAPM05248PA/html/2010/2020/.

Castillo, A. C. (2016). Planificación y diseño de redes FTTH basadas en zonificación y servicios. Universidad de Panamá.

Commoscope. (09 de 2018). Commoscope. Obtenido de https://es.commscope.com

EXFO. (2012). La Guía FTTH PON. Quebec City, Canada. Obtenido de https://www.c3comunicaciones.es/Documentacion/Guia\%20FTTH\%20PON\%20de\%20EXFO \%202013.pdf

Chomycz, B. (2001). Instalación de fribra óptica, Fundamentos, técnicas y aplicaciones. Latacunga.

Cortes, A. (2016). RedesFTTH, tecnologias GPON, planificacion de la red, diseño de la red, red de fibra optica, nodo de acceso. Panama: Texto del articulo Tecnologia a Fondo.

Edison Quisnancela, N. E. (2016). Certificación de redes GPON, normativa ITU G.984.x.

FTTH Council Europe. (2016). Creating a brighter future. Europe: D\&O Committee.

FTTH Council Europe. (2016). FTTH Handbook. Pauline Rigby.

G.Kramer, B. M. (2006). Ethernet PON (EPON): Design and Analysis of a Optical Access Network. New Jersey: John Wiley \& Sons.

Peres, W., Galperin, H., \& Jordán, V. (2010). Acelerando la revolución digital: banda ancha para América Latina y el Caribe. Santiago de Chile.

Inacap, Universidad Técnologica de Chile. (2010). Redes ópticas pasivas. Santiago de Chile.

Khan, T. Z. (2016). Design of Symme tric 10 Gbps Bi-Directional Wavelength Reused Optical Access Networks. Bangladesh.

FTTH Council Europe. (2016). FTTH Handbook. Pauline Rigby.

G.Kramer, B. M. (2006). Ethernet PON (EPON): Design and Analysis of a Optical Access Network. New Jersey: John Wiley \& Sons.

Galeano Corchero, J. (2009). Diseño e instalación de una red FTTH. Madrid .

Laureles, J. (2016). Incremental FTTH deployment planning. Potchefstroom.

UNIÓN INTERNACIONAL DE TELECOMUNICACIONES. (2003). Recomendación UIT-T G.984.1 (Características generales).

UNIÓN INTERNACIONAL DE TELECOMUNICACIONES. (2004). ITU-T G.984 Especificación de la capa de convergencia de transmisión. 
Unitronics . (2015). Nuevos estándares PON para sistemas FTTx. En P. Notario, Redes de Fibra óptica (págs. 62-64).

Villacrés Valverde , J. C., \& Muriel Bonilla , A. G. (2016). Estudio Y Diseño De Unaredde Planta Externa De Fibra Óptica Gpon Para Proveer Servicios De Voz, Video Y Datos Aplicado A La Ciudad De Alausípara La Cnt Empresa Pública Riobamba. Riobamba . 